\title{
Spinal Instrumentation Rescue with Cement Augmentation
}

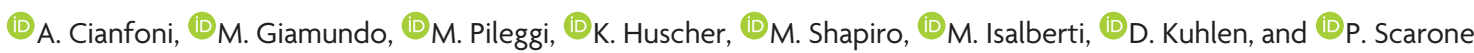

\begin{abstract}
BACKGROUND AND PURPOSE: Altered biomechanics or bone fragility or both contribute to spine instrumentation failure. Although revision surgery is frequently required, minimally invasive alternatives may be feasible. We report the largest to-date series of percutaneous fluoroscopically guided vertebral cement augmentation procedures to address feasibility, safety, results and a variety of spinal instrumentation failure conditions.
\end{abstract}

MATERIALS AND METHODS: A consecutive series of 31 fluoroscopically guided vertebral augmentation procedures in 29 patients were performed to address screw loosening ( 42 screws), cage subsidence (7 cages), and fracture within (12 cases) or adjacent to (11 cases) the instrumented segment. Instrumentation failure was deemed clinically relevant when resulting in pain or jeopardizing spinal biomechanical stability. The main study end point was the rate of revision surgery avoidance; feasibility and safety were assessed by prospective recording of periprocedural technical and clinical complications; and clinical effect was measured at 1 month with the Patient Global Impression of Change score.

RESULTS: All except 1 procedure was technically feasible. No periprocedural complications occurred. Clinical and radiologic follow-up was available in 28 patients (median, 16 months) and 30 procedures. Revision surgery was avoided in 23/28 (82\%) patients, and a global clinical benefit (Patient Global Impression of Change, 5-7) was reported in 26/30 (87\%) cases at 1-month follow-up, while no substantial change (Patient Global Impression of Change, 4) was reported in 3/30 (10\%), and worsening status (Patient Global Impression of Change, 3), in 1/30 (3\%).

CONCLUSIONS: Our experience supports the feasibility of percutaneous vertebral augmentation in the treatment of several clinically relevant spinal instrumentation failure conditions, with excellent safety and efficacy profiles, both in avoidance of revision surgery and for pain palliation.

ABBREVIATIONS: PGIC = Patient Global Impression of Change; PMMA = polymethylmethacrylate

$\mathbf{S}$ pinal instrumentation is widely used in the treatment of degenerative, traumatic, and neoplastic conditions. Altered biomechanics and/or bone fragility may lead to instrumentation failure, bone resorption, or new fractures with consequent instability and recurrent or progressive pain. ${ }^{1,2}$ The most commonly encountered types of instrumentation failure are implant fracture or

Received May 21, 2018; accepted after revision July 23.

From the Departments of Neuroradiology (A.C., M.P., M.I.) and Neurosurgery (M.G., D.K., P.S.), Neurocenter of Southern Switzerland, Ente Ospedaliero Cantonale, Lugano, Switzerland; Department of Neurosurgery (K.H.), Hôpital du Valais, Sion, Switzerland; Department of Radiology (M.S.), New York University Langone Medical Center, New York, New York; and Department of Neuroradiology (A.C.), Inselspital, Bern, Switzerland.

Please address correspondence to Alessandro Cianfoni, PD, MD, Department of Neuroradiology, Neurocenter of Southern Switzerland, Ospedale Regionale di Lugano, Via Tesserete 46, 6900 Lugano, Switzerland; Department of Neuroradiology, Inselspital, Freiburgstrasse 18, 3010 Bern, Switzerland; e-mail: alessandro.cianfoni@eoc.ch

三 Indicates article with supplemental on-line table.

http://dx.doi.org/10.3174/ajnr.A5795 disassembly, bone resorption around the screws, or impaction fracture adjacent to an implanted cage. ${ }^{1}$ In addition, vertebral insufficiency fractures can occur within the instrumented segment or at adjacent levels (junctional fractures). In many instances described above, revision surgery is performed, ${ }^{3}$ with the potential of further morbidity, increased cost, and reduced patient satisfaction. Re-operation is often an unattractive option in elderly, medically complex, and fragile patients.

Minimally invasive options would be desirable to address instrumentation failure. Vertebral cement augmentation is used in the treatment of painful osteoporotic and tumor-related compression fractures. ${ }^{4-7}$ Numerous reports also document the utility of polymethylmethacrylate (PMMA) to augment pedicular screws at the time of insertion. ${ }^{8-12}$

To date, several small series have described the use of cement augmentation in implant failure, including junctional fractures and screw loosening, both in osteoporotic ${ }^{13-15}$ and neoplastic settings. ${ }^{16,17}$ 


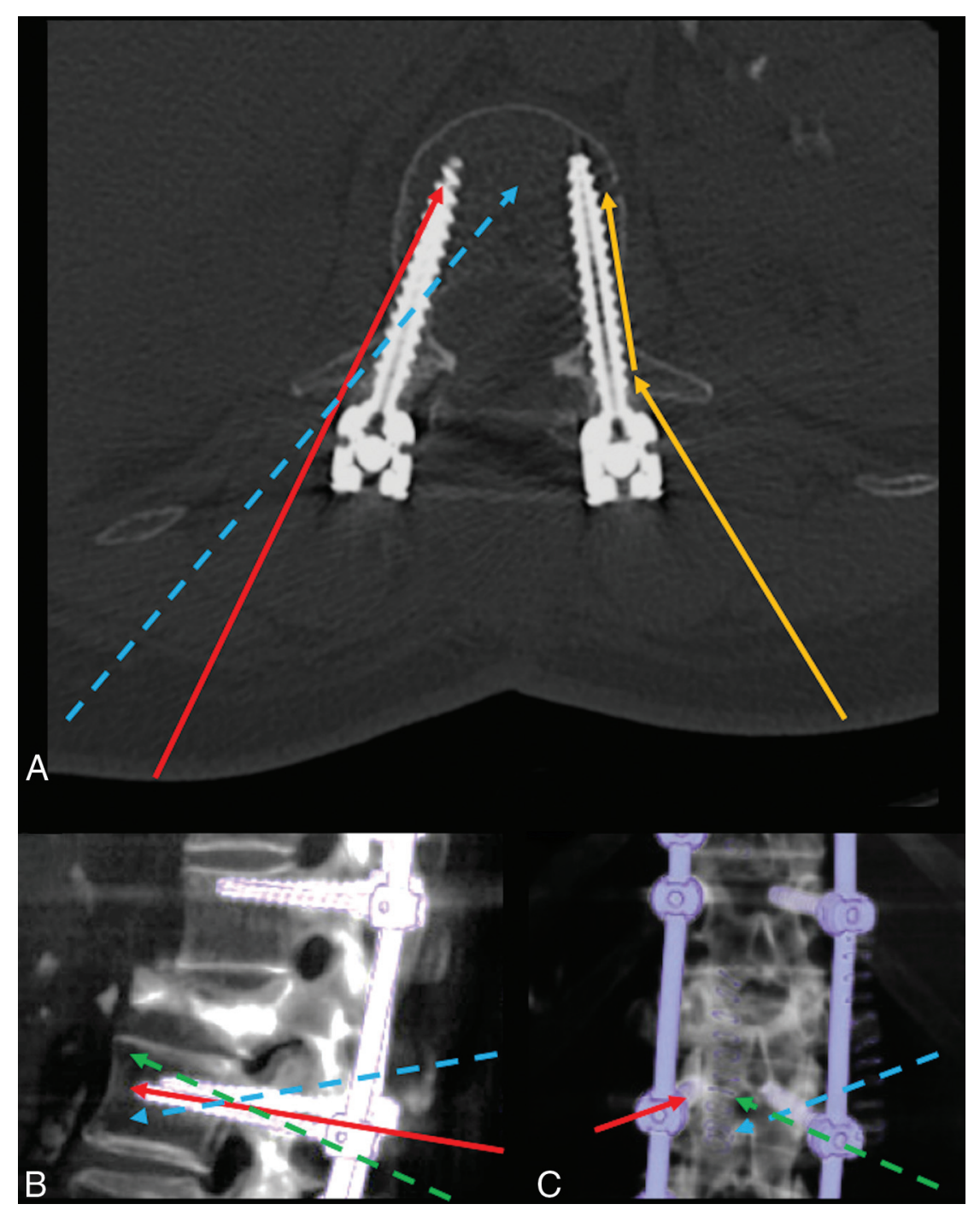

FIG 1. Schematic representation of different access approaches to the instrumented vertebra. $A$, Axial CT image of a vertebra instrumented with bilateral pedicular screws. Yellow arrows represent transpedicular access, with a thin, flexible, beveled needle contacting the proximal screw shaft, then bending and sliding along the screw shaft. The red arrow represents transpedicular access targeting the tip of the screw, with a slightly more oblique course than the screw path. The dashed blue arrow represents extrapedicular access targeting the anterior third of the vertebral body along the midline, crossing the course of the screw at the level of posterior wall with an obliquity from lateral to medial. $B$ and C, Volume-rendered CT lateral and posteroanterior views of an instrumented spine segment. Red arrows in $B$ and $C$ show transpedicular access parallel to the screw used to augment loose screws, while dashed arrows represent extrapedicular accesses to the vertebral body used to augment vertebral body fractures, coursing lateral to medial to the screw, traversing the screw course from cranial to caudal (blue dashed arrows) or from caudal to cranial (green dashed arrow), respectively, passing cranial or caudal to the transverse process.

We report the largest and most encompassing series to date of percutaneous fluoroscopically guided vertebral cement augmentation in a variety of clinically significant instrumentation failure conditions, including screw loosening, cage subsidence, and vertebral fractures within or adjacent to the instrumented segment.

\section{MATERIALS AND METHODS}

A retrospective analysis of 31 consecutive procedures in 29 patients (male/female, 10:19; mean age, 71.6 years; range, 50-82 years) with instrumentation failure treated by percutaneous cement augmentation between May 2013 and October 2016 was performed. Informed consent was obtained from all patients, and the study was approved by the Comitato Etico Cantonale del Ticino institutional ethics committee.

Indications for primary spinal instrumentation included a variety of traumatic, degenerative, and neoplastic conditions. Indications for cement rescue treatment were the following: clinically relevant screw loosening with bone resorption, cage subsidence, and vertebral fracture within or adjacent to the instrumented segment. Instrumentation failure was deemed clinically relevant if accompanied by new or recurrent pain correlated with imaging findings or deemed to be a threat to spinal biomechanical stability. Determination of implant failure, the decision to offer treatment, and the treatment approach were based on case review by the Spine Unit staff of our institution, composed of neurosurgeons, neurologists, neuroradiologists, pain physicians, and physical medicine and rehabilitation physicians. All patients underwent preprocedural noncontrast CT in addition to pre-existing imaging studies. Patients with uncorrectable coagulopathy and local or systemic infection were excluded.

\section{Cement-Augmentation Procedure}

Procedures were performed with the patient under moderate sedation and local anesthesia. A single dose of prophylactic intravenous antibiotic (cefazolin, $2 \mathrm{~g}$ ) was administered 1 hour before the procedure. All procedures were performed under fluoroscopic guidance in a monoor bi-plane angiosuite. A variety of approaches to the target vertebral body were used (Fig 1) to overcome access constraints posed by the presence of implants. Vertebroplasty 15-ga bevel-tip trocars were inserted via a transpedicular or extrapedicular approach to reach the desired target location, and high-viscosity PMMA cement (VertaPlex HV; Stryker, Kalamazoo, Michigan) was injected under real-time fluoroscopic control. Postprocedural target-level CT was performed. Patients were mobilized immediately following recovery from moderate sedation. Most patients were discharged the same day.

\section{Vertebral Body Access}

Transpedicular Approach. An access trajectory parallel to the screw was used for treatment of screw loosening. An en face view of the screw was obtained as a "bull's eye" projection, the fluoroscopic camera was then angled slightly toward the side of the 


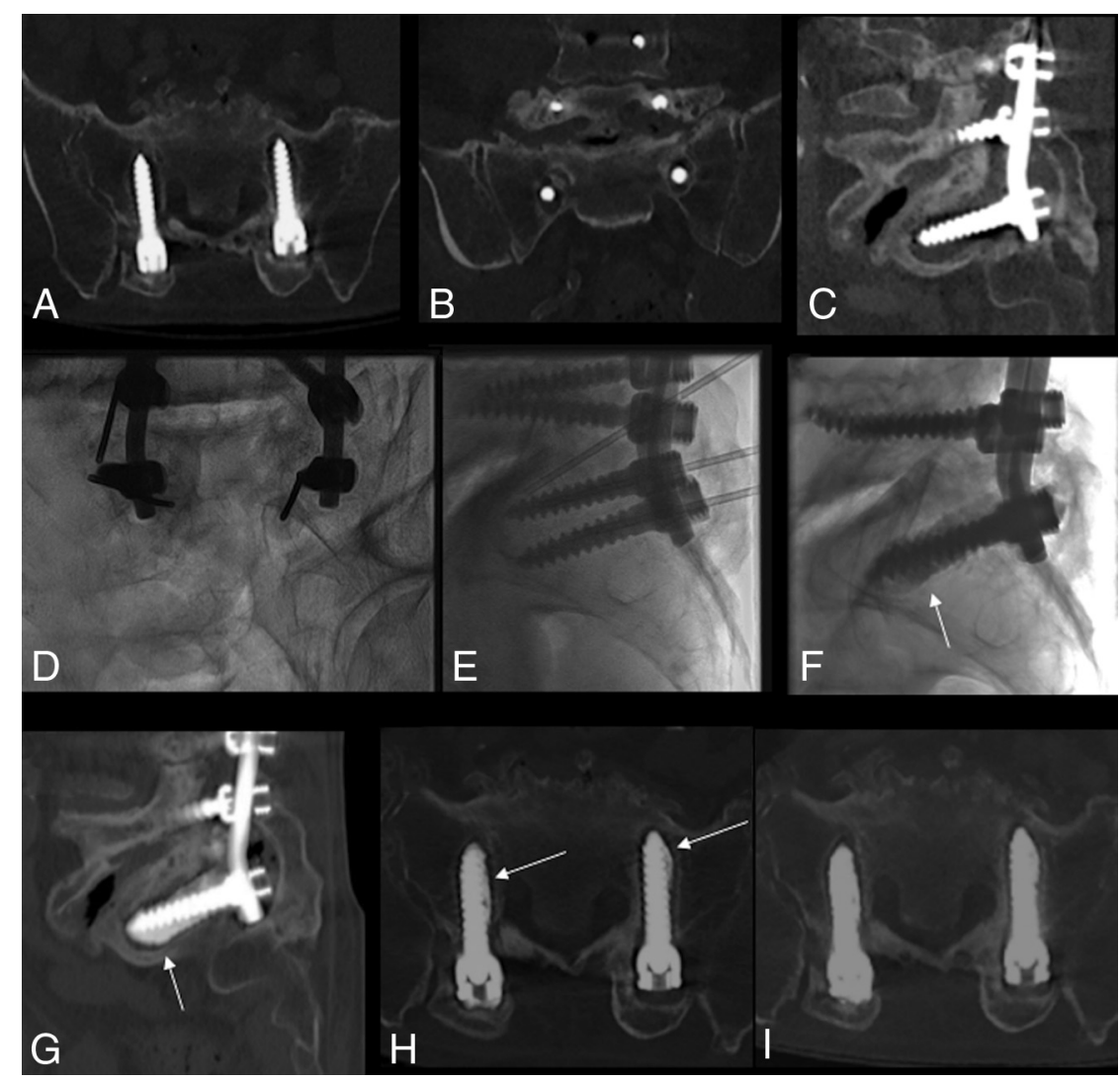

FIG 2. Cement augmentation of bilateral S1 screw loosening. $A-C$, Multiplanar preprocedural CT shows circumferential osteolysis around the screws in S1. Frontal $(D)$ and lateral oblique views $(E)$ of S1 screws, with bilateral placement of needles along the screws and the needle tip in bone osteolysis around the screw. $\mathrm{F}-\mathrm{H}$, Fluoroscopic and CT MIP images post-cement augmentation, demonstrating optimal filling of the osteolytic area (arrows), acting as screw oversizing, and potentially reducing hypermobility. I, Follow-up CT 3 months postaugmentation shows stable results in this patient reporting clinical amelioration.

desired modified access, and the proximal or midportion of the screw was set as the target. With use of a 15-cm 15-ga beveled-tip vertebroplasty needle, contact with the midshaft of the screw was obtained and the flexible needle was pushed to slide with the bevel along the distal shaft of the screw, maintaining the needle tip position inside the bone resorption halo. Alternatively, the tip of the screw was set as the fluoroscopic target on the en face view and aligned with a transpedicular access route a few millimeters away from the screw.

For access to instrumented level fractures (rather than loosening), a trajectory crossing from lateral to medial and from cranial to caudal relative to pedicle screw was usually chosen. In this case, the fluoroscope was angled from the bull's eye view of the screw slightly laterally and cranially. Whenever the needle contacted a screw, appropriately turning the bevel would allow the needle to slide past the screw and continue forward progress to reach the desired target in the vertebral body.

Extrapedicular Access. In general, we thought that small-caliber, straight anteroposterior, and steep craniocaudal orientation of mid and upper thoracic pedicles favored an extrapedicular approach between the pedicle and the rib head. ${ }^{18}$ The access trajectory could be slightly lateral and cranial to the screw and then parallel to the screw, exploiting the bevel design and flexibility of the access needle. For loose screw indications, a similar final nee- dle position was targeted as discussed in the transpedicular section above. For instrumented-level fractures, the approach was to cross the path of the screw at the junction between the pedicle and vertebral body, from lateral to medial, and cranial or caudal to the screw, depending on target fracture location (Fig 3). ${ }^{19}$ In case of access to the vertebral body without pedicular screws, a standard approach was used, with only necessary adjustments to avoid the vertical rods.

Injection of high-viscosity PMMA cement was performed under real-time high-resolution fluoroscopic control, predominantly in the lateral view, with intermittent anteroposterior or oblique control views.

In case of screw loosening (Fig 2), cement injection was aimed at filling the halo of bone resorption around the screw, to simulate screw oversizing, and reducing or nulling screw micromobility. Whenever possible, the adjacent trabecular network was also filled with cement in an effort to achieve a more stable anchoring cast between the screw and the vertebral body. In case of vertebral fracture, cement injection was aimed at filling, with a trabecular interdigitation pattern, the anterior two-thirds of the vertebral body, from superior to inferior endplates on both sides of the midline. In case of cage subsidence, cement injection was aimed at augmenting the bone-metal interface and arresting bone compaction (Fig 3).

\section{Follow-Up}

Patients were followed 1 month after the procedure with a clinical visit and standing plain films; when deemed necessary, CT or MR imaging was performed. Clinical and imaging follow-up was continued at variable intervals, depending on the clinical situation and the referring physician's preference.

\section{Study End Points}

Study end points were feasibility, safety, and efficacy. Feasibility and safety were assessed by review of chart records and periprocedural complications; efficacy was based on the rate of revision surgery, while the clinical effect on pain was measured at 1-month follow-up. The Patient Global Impression of Change (PGIC) 7-point response was scored as follows: 1 , extremely worse; 2 , much worse; 3 , a little worse; 4 , no change; 5 , a little better; 6 , much better; and 7, extremely better. ${ }^{20}$

\section{RESULTS}

The On-line Table summarizes the results.

In 31 procedures, performed in 29 patients ( 2 patients underwent 2 procedures), 42 loose screws, 7 levels with cage subsidence, 


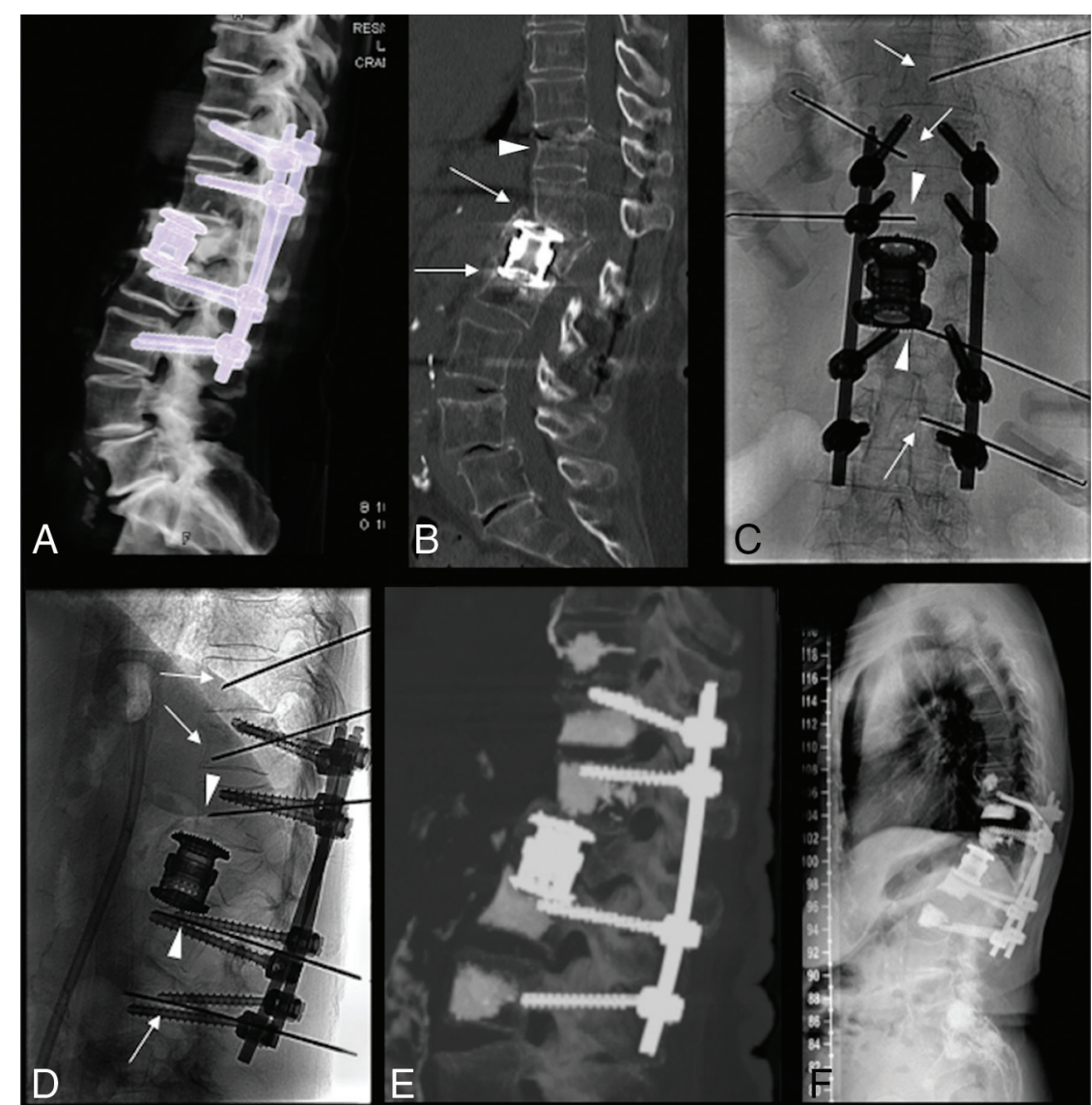

FIG 3. Cage subsidence/fracture and multiple targets. $A$ and $B, C T$ images of an $L 1$ fracture treated with corpectomy, cage grafting, and T11-L3 posterior stabilization in a patient with osteoporosis. Due to bone compaction/fracture cranial and caudal to the cage, there is cage subsidence and focal kyphosis (arrows in B). Another fracture is noted at T11 (arrowhead in B), and there is bone resorption and screw loosening at L3 (not shown), with initial screw pullout. $C$ and $D$, Anteroposterior and lateral fluoroscopy views after placement of multiple needles to perform cement augmentation at the cranial and caudal bone-metal cage interface in 712 and L2 (arrowheads), in the T11 fracture (arrow), in L3 (arrow) to augment the screw osseous purchase, and in T10 to perform prophylactic augmentation (arrow). E, Postprocedural sagittal MIP CT image demonstrates satisfactory cement filling of the target levels. F, Standing plain film at 12-month follow-up, with stable results. major complications ${ }^{21}$ occurred; specifically, there were no neurologic complications or clinically significant PMMA leaks. Clinical and radiologic follow-up at 1 month was available in 28 patients; extended follow-up ranged from 2 to 54 months (median, 16 months; interquartile range, 14.7 months). Specifically, follow-up was available at 1 month in 28 patients, at 3 months in 26, at 6 months in 24 , and beyond 12 months in 18 . One patient was lost to follow-up.

During the follow-up period, 5/28 (18\%) patients required revision surgery: The patient whose procedure was aborted due to access failure underwent revision surgery with replacement of a loose screw; another patient developed re-fracture and kyphosis despite cement augmentation of a junctional fracture requiring extension of instrumentation. Three patients underwent implant removal. In 1 of these 3 patients, instrumentation failure was ultimately due to a low-grade chronic infection.

In the 28 patients (30 procedures) with available follow-up, global clinical benefit (PGIC 5-7) was reported in $26 / 30(87 \%)$ cases at 1 month after the procedure (PGIC 7 in 10/26, PGIC 6 in 9/26, PGIC 5 in 7/26), while no significant changes in status (PGIC 4) were reported in $3 / 30(10 \%)$ and worsening status (PGIC 3) occurred in $1 / 30(3 \%)$ in the absence of obvious procedural complications.
12 vertebral body fractures at levels within the instrumented segment, and 11 fractures at levels adjacent to the instrumented segment (junctional fractures) were treated. In addition, prophylactic cement augmentation was performed at nonfractured adjacent levels in 9 cases with the intent of preventing subsequent junctional fractures in patients with osteoporosis when focal kyphosis was noted at the junctional level. In some patients, during the same procedure, distant vertebral or sacral fractures were also treated with cement augmentation. Prophylactic and distant site augmentation was not included in analysis.

In all 32 thoracic, 24 lumbar, 13 sacral, and 3 pelvic (iliac screws) targets, a total of 72 targets, were treated.

The mean interval between the last spinal instrumentation and the procedure was 14 months (range, 1 week to 11 years; median, 4.5 months).

All except 1 procedure was technically feasible and successfully accomplished (ie, satisfactory fluoroscopic target visibility, needle placement, and cement injection). One procedure was aborted due to access failure caused by implant-related inability to visualize fluoroscopic landmarks. No periprocedural minor or

\section{DISCUSSION}

In this series, minimally invasive fluoroscopically guided percutaneous cement augmentation was associated with successful avoidance of revision surgery in $82 \%$ of patients with screw loosening, cage subsidence, or vertebral fractures within or adjacent to the instrumented segment. Although limited by the retrospective study design, we believe that implant failure would have otherwise led to revision surgery, as discussed in the Spine Unit multidisciplinary review. Technical feasibility was highly satisfactory, with $30 / 31$ procedures executed with the desired technical results. Safety was excellent, with no periprocedural complications, and the procedure resulted in significant pain amelioration in $87 \%$ of cases.

Spinal instrumentation performed for degenerative, traumatic, or oncologic diseases that may cause deformity or instability aims at providing stability while osseous fusion develops. Adequate bone quality is of primary importance in this success. Inadequate fixation and subsequent segmental microinstability may put the implant at risk of ultimate failure. ${ }^{22}$ A combination of 
factors such as initial or subsequent poor bone density/quality, instrumentation-induced stress shielding and subsequent disuse osteopenia, plastic deformation at the bone-metal interface, or high static stress combined with cyclic loading also contribute to instrumentation failure. ${ }^{2}$ These processes are often accompanied by new or recurrent pain, altered biomechanics, and deformity. Implant failure can occur at variable time intervals after instrumentation from weeks to years. To interrupt this vicious spiral and palliate pain, in most cases, revision surgery with screw replacement, cage replacement with the addition of allograft or other bony substitutes, or instrumentation extension are usually necessary. All these options carry perioperative risks and morbidity and are sometimes contraindicated by patients' clinical conditions, posing a therapeutic dilemma; in addition, revision surgery has an important cost burden. ${ }^{23}$ While in cases of major instrumentation failure, such as implant fracture or disassembly, open surgery seems absolutely necessary, in other conditions, there may be room for a minimally invasive treatment option such as cement augmentation. This technique, established for the treatment of vertebral body compression fractures ${ }^{4-7}$ and used to reinforce pedicular screws at the time of insertion, ${ }^{8-12}$ has been reported in small series and case reports as a potential solution in case of implant-related vertebral fractures and screw loosening. ${ }^{13-17}$

\section{Technical Feasibility and Safety}

Despite widespread use of vertebral augmentation, there is a paucity of reports on this technique applied to conditions of instrumentation failure. This might be, in part, related to technical hurdles posed by the presence of implants, skepticism regarding procedure efficacy, and lack of awareness. The presence of dense metallic structures sometimes obscures the visibility of known fluoroscopic landmarks or creates artifacts when CT is used for guidance. Commonly used access routes to the vertebral body, namely the transpedicular approach, are often occupied by implants, and the operator is therefore forced to seek other trajectories within narrow anatomic windows. Finally, after the operation, spinal anatomy can be altered by laminectomies and bony fusion masses.

Both CT and fluoroscopic guidance were described in prior reports. ${ }^{13-17}$ We invariably used fluoroscopic guidance based on careful planning and mandatory preoperative CT. Transpedicular access was favored in the lumbar and lower thoracic spine, while an extrapedicular approach was preferably adopted in the mid and upper thoracic spine. A small-caliber 15-ga beveled-tip vertebroplasty needle was used, allowing steerability and flexibility to precisely reach targets.

Precise needle positioning, use of high-viscosity cement, and use of high-quality fluoroscopic imaging equipment might have contributed to the excellent safety profile of the procedures in our series. Although not used in this series, intraoperative CT, O-arm Multidimensional Surgical Imaging System (Medtronic, Minneapolis, Minnesota), or conebeam CT guidance could be implemented to aid needle insertion. ${ }^{13,17}$ Moreover, when necessary, injection of cement can be directed by the use of a coaxial curved cannula. ${ }^{15}$

\section{Clinical Results}

The main clinical objective of this study was to assess the rate of revision surgery avoidance. In a retrospective study, it is not possible to rule out that in some circumstances, a conservative approach would have been sufficient. Pain palliation was also assessed; however, back pain in this kind of complex patient cohort is an elusive target: It is indeed rather difficult to definitively attribute new or recurrent symptoms to imaging findings of implant failure. We preferred to use the PGIC scale rather than a Visual Analog Scale for pain assessment because, in our opinion, it yields a more global and general assessment of the patient's perception of treatment effectiveness. Among patients with available follow-up, the clinical benefit of the procedure was reported in $26 / 30$ cases. The 1 patient in whom the procedure was aborted due to access failure reported unchanged clinical status and ultimately underwent revision surgery. Two more patients reporting a PGIC 4 and 1 patient with PGIC 3 required revision surgery, while 1 patient reporting initial clinical benefit (PGIC 6) after augmentation of 2 loose screws and a junctional fracture had a delayed recurrent collapse of the junctional level and underwent instrumentation extension. Finally, 1 patient was lost to follow-up and remained unreachable; this patient might have undergone revision surgery at another institution.

In our series of 29 patients with implant failure, it was necessary to treat a total of 72 targets, including loose screws, cage subsidence, and vertebral fractures within or adjacent to the instrumented segment. Our data suggest that an important cause of implant failure is poor bone quality, a systemic problem leading to multilevel breakdown (Fig. 3). We therefore stress the extreme importance of aggressive osteoporosis management in this patient population. ${ }^{24}$

Infrequently, bone resorption around implants can be caused by an infectious process. In case of clinical-radiologic suspicion, biopsies and cultures are recommended before proceeding to cement augmentation. Despite these measures, 1 patient in this series, following revision surgery with implant removal, was diagnosed with a low-grade infection, despite biopsy performed 2 weeks before cement augmentation resulting in cultures negative for infection. This infection was likely present before cement augmentation. Suboptimal sensitivity of spine biopsies for low-grade infections remains a challenge in such cases. ${ }^{25,26}$

\section{Limitations}

Major limitations include the retrospective study design and an intrinsically subjective definition of clinically relevant instrumentation failure. Also recognized is the subjective nature of patient self-assessment. Although this is by far the largest series of its kind to date, some conditions such as cage subsidence are infrequent and considerably more experience is necessary. Given the frequency of spinal instrumentation surgery, it is likely that percutaneous salvage options are currently underused; therefore, greater awareness and prospective investigations are necessary.

\section{CONCLUSIONS}

This series supports the feasibility of safe, efficacious, minimally invasive percutaneous vertebral cement augmentation in the treatment of clinically relevant instrumentation failure, with an 
excellent safety profile and efficacious clinical results in terms of pain palliation and avoidance of revision surgery. A larger prospective cohort will be necessary to determine optimal candidates for this treatment and to provide more generalizable outcome data.

Disclosures: Dominique Kuhlen—UNRELATED: Board Membership: DePuy Synthes Advisory Board.* Pietro Scarone-RELATED: Consulting Fee or Honorarium: Depuy Synthes, Comments: I am a consultant spine surgeon for this company, from which my institution receives fees*. *Money paid to the institution.

\section{REFERENCES}

1. DeWald CJ, Stanley T. Instrumentation-related complications of multilevel fusions for adult spinal deformity patients over age 65: surgical considerations and treatment options in patients with poor bone quality. Spine (Phila Pa 1976) 2006;31(19 Suppl):S144-51 CrossRef Medline

2. Pearson HB, Dobbs CJ, Grantham E, et al. Intraoperative biomechanics of lumbar pedicle screw loosening following successful arthrodesis. J Orthop Res. 2017;35:2673-81 CrossRef Medline

3. Kang SH, Kim KT, Park SW, et al. A case of pedicle screw loosening treated by modified transpedicular screw augmentation with polymethylmethacrylate. J Korean Neurosurg Soc 2011;49:75-80 CrossRef Medline

4. Clark W, Bird P, Gonski P, et al. Safety and efficacy of vertebroplasty for acute painful osteoporotic fractures (VAPOUR): a multicentre, randomised, double-blind, placebo-controlled trial. Lancet 2016; 388:1408-16 CrossRef Medline

5. Klazen CA, Lohle PN, de Vries J, et al. Vertebroplasty versus conservative treatment in acute osteoporotic vertebral compression fractures (Vertos II): an open-label randomised trial. Lancet 2010;376: 1085-92 CrossRef Medline

6. Fourney DR, Schomer DF, Nader R, et al. Percutaneous vertebroplasty and kyphoplasty for painful vertebral body fractures in cancer patients. J Neurosurg 2003;98(1 Suppl):21-30 CrossRef Medline

7. Berenson J, Pflugmacher R, Jarzem P, et al; Cancer Patient Fracture Evaluation (CAFE) Investigators. Balloon kyphoplasty versus nonsurgical fracture management for treatment of painful vertebral body compression fractures in patients with cancer: a multicentre, randomised controlled trial. Lancet Oncol 2011;12:225-35 CrossRef Medline

8. Amendola L, Gasbarrini A, Fosco M, et al. Fenestrated pedicle screws for cement-augmented purchase in patients with bone softening: a review of 21 cases. J Orthop Traumatol 2011;12:193-99 CrossRef Medline

9. Sawakami K, Yamazaki A, Ishikawa S, et al. Polymethylmethacrylate augmentation of pedicle screws increases the initial fixation in osteoporotic spine patients. J Spinal Disord Tech 2012;25:E28-35 CrossRef Medline

10. Burval DJ, McLain RF, Milks R, et al. Primary pedicle screw augmentation in osteoporotic lumbar vertebrae: biomechanical analysis of pedicle fixation strength. Spine (Phila Pa 1976) 2007;32:1077-83 CrossRef Medline

11. Chang MC, Liu CL, Chen TH. Polymethylmethacrylate augmenta- tion of pedicle screw for osteoporotic spinal surgery: a novel technique. Spine (Phila Pa 1976) 2008;33:E317-24 CrossRef Medline

12. Fransen $P$. Increasing pedicle screw anchoring in the osteoporotic spine by cement injection through the implant: technical note and report of three cases. J Neurosurg Spine 2007;7:366-69 CrossRef Medline

13. Amoretti N, Bertrand AS, Gallo G, et al. Percutaneous consolidation of loosened spine arthrodesis under CT and fluoroscopy guidance by radiologists: a new useful technique. Eur Radiol 2015 25:1135-39 CrossRef Medline

14. Yun DJ, Hwang BW, Oh HS, et al. Salvage percutaneous vertebral augmentation using polymethyl methacrylate in patients with failed interbody fusion. World Neurosurg 2016;95:618.e13-618.e20 CrossRef Medline

15. Clerk-Lamalice O, Irani Z, Growney M, et al. Parapedicular vertebral augmentation with polymethylmetacrylate for pedicle screw loosening. J Neurointerv Surg 2018 Feb 14. [Epub ahead of print] CrossRef Medline

16. Puri AS, Erdem E. Salvage percutaneous vertebral augmentation in failed spinal interbody fusions associated with multiple myeloma. Spine J 2010;10:e5-10 CrossRef

17. Xu R, O'Connor K, Krol G, et al. Cement salvage of instrumentation-associated vertebral fractures. AJNR Am J Neuroradiol 2014;35: 2197-201 CrossRef Medline

18. Cianfoni A, Boulter DJ, Rumboldt Z, et al. Guidelines to imaging landmarks for interventional spine procedures: fluoroscopy and CT anatomy. Neurographics 2011;1;39-48 CrossRef

19. Cianfoni A, Massari F, Ewing S, et al. Combining percutaneous pedicular and extrapedicular access for tumor ablation in a thoracic vertebral body. Interv Neuroradiol 2014;20:603-08 CrossRef Medline

20. Hurst H, Bolto J. Assessing the clinical significance of change scores recorded on subjective outcome measures. J Manipulative Physiol Ther 2004;27:26-35 CrossRef Medline

21. Radvany MG, Murphy KJ, Millward SF, et al; Technology Assessment Committee of the Society of Interventional Radiology. Research reporting standards for percutaneous vertebral augmentation. J Vasc Interv Radiol 2009;20:1279-86 CrossRef Medline

22. Young PM, Berquist TH, Bancroft LW, et al. Complications of spinal instrumentation. Radiographics 2007;27:775-89 CrossRef Medline

23. Theologis AA, Miller L, Callahan M, et al. Economic impact of revision surgery for proximal junctional failure after adult spinal deformity surgery: a cost analysis of 57 operations in a 10-year experience at a major deformity center. Spine (Phila Pa 1976) 2016;41: E964-72 CrossRef Medline

24. Ohtori S, Inoue G, Orita S, et al. Comparison of teriparatide and bisphosphonate treatment to reduce pedicle screw loosening after lumbar spinal fusion surgery in postmenopausal women with osteoporosis from a bone quality perspective. Spine (Phila Pa 1976) 2013;38:E487-92 CrossRef Medline

25. Prodi E, Grassi R, Iacobellis F, et al. Imaging in spondylodiskitis. Magn Reson Imaging Clin N Am 2016;24:581-600 CrossRef Medline

26. Zimmerli W. Clinical practice: vertebral osteomyelitis. N Engl J Med 2010;362:1022-29 CrossRef Medline 